Research Paper

\title{
Thyroid-Stimulating Hormone Is Significantly Associated with Bone Health Status in Men
} \author{
api Johari ${ }^{3}$, and Wan Zurinah Wan Ngah $^{3}$ \\ 1. Pharmacology Department, Faculty of Medicine, Universiti Kebangsaan Malaysia. \\ 2. Physiology Department, Faculty of Medicine, Universiti Kebangsaan Malaysia. \\ 3. Biochemistry Department, Faculty of Medicine, Universiti Kebangsaan Malaysia.
}

Kok-Yong Chin ${ }^{1}$, Soelaiman Ima-Nirwana ${ }^{\circledR}$, Isa Naina Mohamed ${ }^{1}$, Amilia Aminuddin², Mohamad Han-

$\triangle$ Corresponding author: Professor Dr. Soelaiman Ima-Nirwana, M.B.B.S., PhD. Department of Pharmacology, Faculty of Medicine, Universiti Kebangsaan Malaysia. Jalan Raja Muda Abdul Aziz, 50300 Kuala Lumpur, Malaysia. Tel: 03-40405514 Fax: 03-26938205 Email: imasoel@medic.ukm.my.

( ) Ivyspring International Publisher. This is an open-access article distributed under the terms of the Creative Commons License (http://creativecommons.org/ licenses/by-nc-nd/3.0/). Reproduction is permitted for personal, noncommercial use, provided that the article is in whole, unmodified, and properly cited.

Received: 2013.0I.12; Accepted: 2013.03.18; Published: 2013.05.14

\begin{abstract}
Background and Aim: Recent studies revealed a novel association between thyroid-stimulating hormone (TSH) and bone health status in healthy male populations. The present study aimed to validate this association and provide new information on the relationship between TSH levels and calcaneal speed of sound (SOS) in men.

Methods: This cross-sectional study recruited $68 \mathrm{I}$ men with complete data of calcaneal SOS, body anthropometry, serum TSH, free triiodothyronine (FT3) and free thyroxine (FT4) levels.

Results: All subjects had FT3 and FT4 levels within the in-house reference range and I 3 subjects had lower than normal TSH levels. The results revealed that the SOS value of subjects was significantly associated with TSH after multiple adjustments $(p<0.05)$. When subjects were divided into quintiles according to their TSH levels, the difference of SOS between men with low-normal TSH and high-normal TSH contributed significantly to the association between TSH and bone health status $(\mathrm{p}<0.05)$. The significance of the association persisted with the inclusion and exclusion of subclinical hyperthyroid subjects.

Conclusions: There was a significant association between TSH levels and bone health status in men as assessed by quantitative ultrasound. This age-independent association between TSH and SOS might explain some of the individual variation of bone health status in men.
\end{abstract}

Key words: Thyroid-stimulating hormone, bone, calcaneus, men.

\section{Introduction}

It has been well-established that overt hyperthyroidism is a risk factor for osteoporosis (1). The observation of low bone health status among patients with subclinical hyperthyroidism, a condition defined by normal thyroid status but suppressed thyroid-stimulating hormone (TSH) level, evokes speculation that TSH may have a direct effect on bone health status apart from its traditional role as a regulator of thyroid hormones (2-4). In a cross-sectional study, postmenopausal women with low TSH levels were found to have significantly lower femoral hip and lumbar spine bone mineral density (BMD) and higher levels of bone remodeling markers compared to women with normal TSH levels (5). Nevertheless, the prevalence of subclinical hyperthyroidism is low in the general population (6). The association of TSH with bone health status in a healthy euthyroid population is a more intriguing research question because 
it may explain part of the individual variation of bone health status in men.

The evidence for the association between TSH and bone health status is growing. Thyroid-stimulating hormone receptor (TSHR) has been found in bone cells (7). In TSHR knockout rodents, severe osteoporosis associated with increased osteoclastogenesis was observed even when they were given thyroid supplementation (8). Intermittent TSH administration was shown to prevent and restore bone health in rodents $(9,10)$. In some epidemiological studies, TSH levels were found to be associated with bone mineral density (BMD) in healthy euthyroid males and post-menopausal females $(11,12)$. In these studies, the association of TSH with BMD was limited to subjects with high-normal and low-normal TSH levels $(11,12)$. There were also studies reporting no significant association between bone health status and TSH in subjects with normal TSH levels $(13,14)$. Hence, the significance of this relationship needs to be validated in other populations. Besides, many of these studies focused at females, whereas the relationship between TSH and bone health was studied less in men.

The current study was aimed to investigate the association between TSH levels and bone health status in healthy Malaysian men aged 20 years and above. We assessed bone health status in the current study population using the calcaneal quantitative ultrasound technique because previous studies had used DXA to examine the relationship between TSH levels and bone health status in men $(11,13,15)$. There was one study which used amplitude-dependent speed of sound (Ad-SOS) at the proximal phalange to assess the bone health status in pre- and postmenopausal women with and without subclinical hyperthyroidism (16). However, the sample size of the study was small and included only female subjects. Besides, proximal phalange was not a recognized site for quantitative ultrasound assessment of bone (17). No studies have been performed on healthy males using the quantitative ultrasound method. This was a part of our continuous efforts to examine various factors that could influence quantitative ultrasound indices in men $(18,19)$. We hoped this would generate information on the role of TSH as a potential endogenous factor associated with individual variations in bone health status in men.

\section{Materials and Methods}

\section{Study Design}

This cross-sectional study was conducted as part of the Malaysian Aging Male Study, which aimed at assessing the general health status of Malaysian men, specifically in the Klang Valley $(19,20)$. Subjects were recruited via purposive sampling from September 2009 to September 2011. Recruitment was conducted through advertisements in major newspapers, radio broadcasts, flyers, public announcements in community centres and religious places. The study protocol was reviewed and approved by the Ethics Committee of Universiti Kebangsaan Malaysia Medical Centre. All subjects were briefed in details information regarding this study and they provided written consent before participating in this study.

\section{Subjects}

The subjects recruited were Chinese and Malay men aged $\geq 20$ years residing in the Klang Valley (Kuala Lumpur and its surrounding areas), Malaysia, who had visited our screening facility throughout the study period. They answered a questionnaire on basic demographic details and medical status. The age of the subjects was obtained from their identification cards and their ethnicity was self-declared. History taking and physical examination were performed by qualified physicians. Subject also underwent body anthropometry and calcaneal speed of sound (SOS) measurements, and blood collection. Subjects with the following conditions were excluded from the study: 1) those taking drugs known to affect bone metabolism, such as thyroxine, testosterone, corticosteroids, antidepressants etc.; 2) those suffering from chronic diseases known to cause changes in bone health status, such as hyper/hypoparathyrodism, hyper/ hypocalcemia, Paget's disease, osteogenesis imperfecta, osteomalacia and osteoporosis; 3) those suffering from impaired mobility and needing a walking aid; 4) those who suffered from a fracture within six months prior to the screening session; 5) those who were unable to complete the screening process.

\section{Calcaneal speed of sound measurement}

The calcaneal speed of sound measurement was conducted using the Furuno CM-200 sonometer (Furuno Electric, Nishinomiya City, Japan). It was a gel-based system which generated speed of sound (SOS) as the determinant of bone health status. In general, a higher SOS indicated better bone health status. The subjects were required to sit on a chair and place their right feet on the foot pad so that their calcaneus was in close contact with both the transducers on its lateral sides. Upon initiation, the ultrasound wave would propagate from one transducer to the other through the calcaneal bone. Each subject was measured three times and the average values were taken as the SOS values for the respective subjects. All 
measurements were performed by a trained technician using a single machine. Calibration was conducted prior to each screening session. The short-term in-vivo coefficient of variation (CV) value for CM-200 calculated (as per instructions of Bonnick et al (21)) was $0.19 \%(18)$.

\section{Body anthropometry measurement}

The weight of subjects with light clothing but without shoes was determined using a weighing scale (Tanita, Tokyo, Japan) and was recorded to the nearest $0.1 \mathrm{~kg}$. The height of the subjects without shoes was measured using a portable stadiometer (Seca, Hamburg, Germany) and was recorded to the nearest $0.1 \mathrm{~cm}$. The body mass index (BMI) of the subjects was calculated using the formula: $\mathrm{BMI}=$ (height of subject in meter $)^{2}$ / (body weight of the subject in $\mathrm{kg}$ ).

\section{Hormonal measurement}

Venous blood of the subjects was collected between 0830 and 1030 during each screening session after an overnight fast. Phlebotomy was performed by a qualified physician or phlebotomist. Serum was extracted immediately and stored at $-70^{\circ} \mathrm{C}$ until analysis. Free triiodothyronine (FT3), free thyroxine (FT4) and TSH were determined using an ADVIA Centaur (Siemens Healthcare Diagnostics, Illinois, USA) based on competitive immunoassay with direct chemiluminescent technology. Inter-assay CV for FT4 was 5.34-11.50\% (in-house reference: 9.0-25.0 pmol/1), FT3 was $1.46-2.57 \%$ (in-house reference: $3.5-6.5 \mathrm{pmol} / \mathrm{l}$ ) and TSH was $0.37-3.37 \%$ (in-house reference: $0.40-4.70$ $\mathrm{mIU} / \mathrm{l})$

\section{Data analysis}

Bivariate Pearson's correlation analysis was performed to assess the association between FT3, FT4, TSH and SOS with age, body anthropometry and ethnicity to identify potential confounding factors. The characteristics of the Chinese and Malay subjects were compared using univariate analysis with necessary adjustments for confounders. Multiple regression analysis was conducted to assess the relationship between TSH (as a continuous variable) and SOS, with adjustments for FT3 and FT4 levels, thus forming Model 1. The model was further adjusted for age (Model 2), body anthropometry (Model 3) and ethnicity (Model 4). Changes in standardized regression $(\beta)$ value were observed. Multivariate normality was ensured by eliminating standardized residual of regression model greater than 2.0. Normality of the standardized residual was tested using the Shapiro-Wilk test with the aid of a histogram. Next, the TSH levels were divided into quintiles (Q1-Q5) and entered into the multiple regression models to assess the contribution of the differences between Q1 and Q2-Q5 towards the association between TSH and SOS. This was performed to validate the previous finding which showed that the difference between low-normal (Q1) and high-normal (Q5) TSH was responsible for most of the associations between TSH and SOS $(11,12)$. The significant value for all analysis was set at $p<0.05$. The analysis was performed using the Statistical Package for Social Sciences (SPSS) version 16.0 (SPSS Inc., Chicago, USA).

\section{Results}

A total of 840 men visited our screening facility, from which 769 subjects consented to blood collection. After eliminating subjects without calcaneal SOS $(n=8)$ and body anthropometry measurements $(n=8)$, 753 subjects remained. After removing univariate and multivariate outliers, the number of subjects used in the final analysis was 681 men $(81.1 \%$ of the total recruitment), with a mean age of 48.5 years (range: $20-83$ years). The subjects consisted of $45.5 \%$ Malay men and $54.5 \%$ Chinese men.

All subjects had FT3 and FT4 levels within the in-house normal range, while $98.1 \%$ of the subjects had TSH levels within the in-house normal range and $1.9 \%$ of the subjects had TSH levels below the normal range.

Bivariate correlation analysis revealed that there were significant associations $(p<0.05)$ between SOS with age, BMI and ethnicity; TSH with age; FT3 with age, BMI and weight; FT4 with age. These associations indicated possible confounding effects of age, ethnicity and body anthropometry on the variables of interest and they were adjusted for the following analysis (Table 1).

Univariate analysis indicated that there were significant differences in age $(p=0.009)$, weight $(p=0.003)$, height $(p<0.001)$ and BMI $(p<0.001)$ between Chinese and Malay men. The Chinese subjects were older, taller, and lower in body weight and BMI compared to Malay subjects. After adjustment for confounding factors, no significant differences were found in SOS $(p=0.258)$, FT3 $(p=0.197)$, FT4 $(p=0.186)$ and TSH $(p=0.365)$ between Chinese and Malay subjects (Table 2).

Multiple regression analysis revealed that TSH was significantly and positively associated with SOS after adjustment for age $(p<0.05)$. The significance of this association persisted even after further adjustment for body anthropometry and ethnicity $(p<0.05)$. Free T3 and Free T4 were not associated with SOS significantly in all models generated. These findings were observed both in analyses that included (Table 
3A) and excluded (Table 3B) the subjects with lower than normal TSH levels. However, the standardized regression coefficient in models excluding subclinical hyperthyroid subjects was reduced and the associations became marginally significant.

Subjects were divided into TSH quintiles to assess the contribution of the differences in TSH levels towards SOS in multiple regression models. The results showed that only the differences between subjects in Q1 and Q5 were associated significantly with the variation in SOS $(p<0.05)$. This was observed in analyses that included and excluded subclinical hyperthyroid subjects. In further analysis, subclinical hyperthyroid subjects were categorized in a separate group and the subjects with normal TSH were divided into quintiles. The differences in TSH levels between subjects with lower than normal TSH levels and subjects with normal TSH levels were not associated significantly with variation in SOS (Table 4).
Table I. Correlational analysis between demographic and body anthropometry and variables of interest.

\begin{tabular}{llllll}
\hline Variable & Age & Height & Weight & BMI & Ethnicity \\
\hline SOS & $-0.283^{* *}$ & -0.024 & 0.065 & $0.076^{*}$ & $-0.095^{*}$ \\
TSH & $0.152^{* *}$ & 0.015 & 0.062 & 0.052 & 0.064 \\
FT4 & $-0.192^{* *}$ & 0.012 & -0.063 & -0.074 & -0.069 \\
FT3 & $-0.271^{* *}$ & 0.072 & $0.108^{* *}$ & $0.087^{*}$ & -0.007 \\
\hline
\end{tabular}

Abbr: SOS=speed of sound; TSH= thyroid stimulating hormone; FT3=free tri-iodothyronine; FT4=free thyroxine. *indicates significance value of $\mathrm{p}<0.05$ and ${ }^{* *}$ indicates $\mathrm{p}<0.001$.

Table 2. Characteristics of the subjects.

\begin{tabular}{|c|c|c|c|c|}
\hline Variable & $\begin{array}{l}\text { Malay } \\
(\mathrm{n}=310)\end{array}$ & $\begin{array}{l}\text { Chinese } \\
(\mathrm{n}=371)\end{array}$ & $\begin{array}{l}\text { Overall } \\
(\mathrm{n}=681)\end{array}$ & $\mathrm{p}$ \\
\hline Age (years) & $47.0(16.0)$ & 49.8 (11.7) & $48.6(13.9)$ & 0.009 \\
\hline Height (cm) & $165.8(6.5)$ & $168.3(6.2)$ & $167.2(6.4)$ & $<0.001$ \\
\hline Weight (kg) & $71.3(13.7)$ & $68.4(10.9)$ & $69.7(12.4)$ & 0.003 \\
\hline BMI (kg/m2) & $25.9(4.53)$ & $24.1(3.48)$ & $24.9(4.09$ & $<0.001$ \\
\hline $\begin{array}{l}\text { Calcaneal SOS } \\
(\mathrm{m} / \mathrm{s}) \dagger\end{array}$ & $1521.0(27.5)$ & $1515.8(26.9)$ & $1518.2(27.3)$ & 0.258 \\
\hline TSH (mIU/l)†† & $1.37(0.61)$ & $1.44(0.60)$ & $1.41(0.61)$ & 0.197 \\
\hline FT4 (pmol/l)†† & 15.98 (1.89) & $15.72(1.77)$ & $15.84(1.82)$ & 0.186 \\
\hline FT3 (pmol/l)††† & $5.11(0.46)$ & $5.10(0.46)$ & $5.11(0.46)$ & 0.365 \\
\hline
\end{tabular}

Table 3. Multiple regression analysis using TSH and thyroid hormones as predictors of calcaneal speed of sound, (A) with the inclusion subclinical hyperthyroid subjects. B) excluding subclinical hyperthyroid subjects.

\begin{tabular}{|c|c|c|c|c|c|c|c|c|}
\hline Model & Model 1 & & Model 2 & & Model 3 & & Model & \\
\hline & $\beta$ & $\mathrm{p}$ & $\beta$ & $\mathrm{p}$ & $\beta$ & $\mathrm{p}$ & $\beta$ & $\mathrm{p}$ \\
\hline \multicolumn{9}{|l|}{$\mathbf{A}$} \\
\hline TSH & 0.047 & 0.222 & 0.081 & 0.031 & 0.083 & 0.025 & 0.084 & 0.025 \\
\hline FT4 & 0.063 & 0.116 & 0.031 & 0.423 & 0.036 & 0.349 & 0.035 & 0.364 \\
\hline FT3 & 0.042 & 0.300 & -0.026 & 0.514 & -0.043 & 0.280 & -0.041 & 0.295 \\
\hline \multicolumn{9}{|l|}{ B } \\
\hline TSH & 0.041 & 0.291 & 0.075 & 0.047 & 0.077 & 0.041 & 0.077 & 0.040 \\
\hline FT4 & 0.057 & 0.163 & 0.029 & 0.464 & 0.033 & 0.391 & 0.033 & 0.406 \\
\hline FT3 & 0.042 & 0.296 & -0.024 & 0.554 & -0.041 & 0.308 & -0.040 & 0.323 \\
\hline
\end{tabular}

Abbr: TSH= thyroid stimulating hormone; FT3=free tri-iodothyronine; FT4=free thyroxine; $\beta=$ standardized coefficient of variation. P-value in bold indicates a significant association between the variable tested and SOS value.

Model 1 is adjusted for FT3 and FT4. Model 2 is adjusted for variables in model 1 and age. Model 3 is adjusted for variables in model 2 and BMI. Model 4 is adjusted for variables in model 3 and ethnicity. 
Table 4. Multiple regression models when subjects were divided into quintiles according to their TSH levels.

\begin{tabular}{|c|c|c|c|c|c|c|c|c|c|}
\hline & \multicolumn{3}{|l|}{ Model 1} & \multicolumn{3}{|l|}{ Model 2} & \multicolumn{3}{|l|}{ Model 3} \\
\hline & $\begin{array}{l}\text { TSH range } \\
(\mathrm{mIU} / \mathrm{l})\end{array}$ & $\beta$ & $\mathrm{p}$ & $\begin{array}{l}\text { TSH range } \\
(\mathrm{mIU} / \mathrm{l})\end{array}$ & $\beta$ & $\mathrm{p}$ & $\begin{array}{l}\text { TSH range } \\
(\mathrm{mIU} / \mathrm{l})\end{array}$ & $\beta$ & $\mathrm{p}$ \\
\hline Subclinical hyperthyroid & \multicolumn{3}{|c|}{ combined with Q1 } & $0.11-0.40$ & \multirow{2}{*}{\multicolumn{2}{|c|}{$\begin{array}{l}\text { excluded from analysis } \\
\text { reference group }\end{array}$}} & $0.11-0.40$ & \multicolumn{2}{|c|}{ reference group } \\
\hline Q1 & $0.11-0.88$ & \multicolumn{2}{|c|}{ reference group } & $0.40-0.91$ & & & $0.40-0.91$ & -0.017 & 0.761 \\
\hline Q2 & $0.88-1.18$ & -0.006 & 0.895 & $0.91-1.20$ & -0.02 & 0.672 & $0.91-1.20$ & 0.015 & 0.893 \\
\hline Q3 & $1.18-1.48$ & 0.036 & 0.429 & $1.20-1.49$ & 0.036 & 0.437 & $1.20-1.49$ & 0.069 & 0.526 \\
\hline Q4 & $1.48-1.92$ & 0.049 & 0.289 & $1.49-1.92$ & 0.046 & 0.320 & $1.49-1.92$ & 0.08 & 0.468 \\
\hline Q5 & $1.92-3.14$ & 0.101 & 0.029 & $1.92-3.14$ & 0.096 & 0.042 & $1.92-3.14$ & 0.129 & 0.242 \\
\hline
\end{tabular}

Abbr: TSH= thyroid stimulating hormone; $\beta=$ standardized coefficient of variation. P-value in bold indicates a significant association between the variable tested and SOS value. All models (1, 2 and 3) were adjusted for age, BMI and ethnicity.

\section{Discussion}

This study found a significant positive association between TSH levels and calcaneal SOS value in a group of Malaysian males, before and after the exclusion of subjects with subclinical hyperthyroidism. The significance of this positive association persisted when TSH was analysed either as a continuous or as a categorical variable. There was no significant association between free thyroid hormones and bone health in the study population. These results were similar to some studies using DXA as the determinant of bone health. Kim et al. (11) found that lumbar spine BMD was correlated with TSH levels in a group of euthyroid Korean men. Subjects in the lower TSH quintiles had lower BMD compared to subjects in the higher TSH quintiles. In a separate study by Kim et al. (12), similar results were observed in post-menopausal Korean women, in which subjects having low TSH levels (Q1) had 2.2 folds risk of suffering from osteoporosis as compared to subjects having high TSH levels (Q5). The inclusion of subclinical hyperthyroid subjects in the analysis did not significantly alter the results. In the Tromso study, Grimnes et al. (15) observed that TSH levels were associated with BMD in both men and post-menopausal women. Significant associations between TSH and BMD at the distal forearm were only observed in subjects below the 2.5th percentile and above the 97.5th percentile. There was no association between TSH and BMD across the normal range of TSH. In the third National Health and Nutrition Examination Survey of the United States, Morris et al. (22) also found that post-menopausal women in the highest TSH quintile had significantly higher total hip and intertrochanter BMD compared to subjects in the lowest TSH quintile.

Nevertheless, there were also some studies reporting results that were different from the current study. Lin et al. (13) found that total T4 but not TSH was associated with BMD at the wrist in a group of
Taiwanese middle-aged euthyroid males. Roef et al. (14) observed that in 677 healthy young male siblings, there was no significant association between TSH and DXA and pQCT measurements. In the same study, they also found that free and total thyroid hormones and thyroid binding globulin were significantly and inversely associated with BMD, BMC, cortical and trabecular area. In a six-year longitudinal study by Murphy et al. (23), TSH was not associated with BMD at the hip and lumbar spine in healthy euthyroid women, although higher TSH levels were shown to be protective against fracture. We suggested that these discrepancies might arise due to intrinsic differences in the population studied. A study by Van Der Deure et al. (24) showed that TSH receptor (TSHR) polymorphism could explain some of the individual variations in BMD. The difference in the prevalence of TSHR alleles protective to bone health among the study population could alter the significance of the association between TSH and bone health. However, this remained speculative and further studies should be conducted to prove it.

The current study measured bone health via calcaneal quantitative ultrasound technique. The calcaneal region consisted of $90-95 \%$ trabecular bone (25). Trabecular bone was found to be highly porous compared to cortical bone. Its porosity was translated to a large surface area per volume ratio, which allowed stimuli to be exposed to larger bone area (26). In an experimental study by Sun et al. (10), ovariectomized rats given intermittent recombinant TSH injection showed significant improvement in trabecular number and trabecular separation but not in cortical thickness. This implied that trabecular bone was more responsive to the variation in TSH levels in the body compared to cortical bone. Hence, measuring the trabecular-rich calcaneal region would better reflect the association of TSH with bone health.

A total of 13 subjects with subclinical hyperthyroidism were found in this study. None of these subjects were on thyroid suppressive therapy as declared. 
We generated various multiple regression models to assess the effects of including and excluding these subjects in the analysis. Excluding these subjects from the analysis caused the significance of the association between TSH and SOS to shrink, yet it was still statistically significant (Table 3). This showed that subjects with lower than normal and low-normal TSH levels were associated with low SOS compared to those with high-normal TSH levels. However, when TSH was analysed as a categorical variable, the difference between subclinical hyperthyroid subjects and subjects with high-normal TSH (Q5) was not significantly associated with SOS, although the standardized regression value was relatively high (Table 4 ). This was due to a small number of subjects with subclinical hyperthyroidism, which rendered the comparison having small power and thus not significant statistically.

In the models without adjustment for age, no significant association was found between TSH and SOS in the subjects (Table 3 ). We suggest that the association was masked by the effects of age on both variables. Based on the results of correlation analysis, the level of TSH increased with age while SOS values decreased in the study population (Table 1). Similar observations on age-dependent changes in TSH and SOS were demonstrated by previous studies $(20,27)$. The direct association between TSH and SOS was shown after the confounding effect of age was removed. This indicated that the relationship between TSH and SOS was age-independent. Besides, in further studies examining the relationship between TSH levels and SOS, age was a necessary confounding factor which should be adjusted.

The relatively small standardized regression coefficient and p-value indicated that although TSH levels contributed to the variance of SOS in men, there were other more important factors in determining the bone health status of the study population. We demonstrated previously that various factors such as age, body anthropometry, physical activity level and testosterone levels were predictors of SOS values in men $(18,19)$. Despite this, TSH levels still provided additional information in explaining the age-independent variance of bone health status in men.

A remarkable study by Abe et al. (8) demonstrated that TSHR knockout (TSHR $/$-) mice had severely reduced BMD as compared to wild type and supplementation of thyroid extract did not normalize the BMD. They also showed that TSH influenced the formation, resorption and survival of osteoclasts via inhibition of JNK/c-jun and NFKB signalling. It also inhibited bone remodelling by suppressing osteoblast differentiation via inhibition of LRP-5 and Flk-1 ex- pression. Thyroid-stimulating hormone also exerted its antiresorptive effects by mediating the level of tumour necrosis factor alpha (28). A recent study by Baliram et al. (29) demonstrated that TSH stimulated osteoblastogenesis in murine embryonic stem cells via activation of protein kinase $C \delta$ and up-regulation of Wnt5a. More intensive research would be required to confirm the mechanism of the effects of TSH on bone.

This study suggests that TSH, a pituitary hormone, may have a direct influence on bone health status in men apart from its traditional role of regulating thyroid hormones in the body. This encourages a re-examination of the physiological functions of pituitary hormones. Apart from that, TSH may also be a viable treatment option for osteoporosis. Studies by Sumpath et al. (9) demonstrated that TSH was able to prevent and restore osteoporosis in ovariectomized Sprague-Dawley rats at low doses. More importantly, serum T3 and T4 levels in these rats were not increased at these doses. Sun et al. (10) later showed that intermittent administration of TSH at an interval of two weeks in ovariectomized rats displayed powerful antiresorptive effects and the effects lasted for four weeks after termination of the regime as assessed by femoral BMD. In postmenopausal women with thyroidectomy, acute administration of recombinant TSH was accompanied by a suppression of C-telopeptide of type- 1 collagen and an increase of bone alkaline phosphatase, hence displaying the antiresorptive effect of TSH (30). However, there was a paucity of equivalent studies performed on male rodent models of osteoporosis, as well as long-term intervention studies on euthyroid osteopenic or osteoporotic patients. Thus, we were not sure of the effectiveness of TSH intervention on bone health status in humans.

There are several limitations pertaining to this study. A non-randomized sampling method was adopted due to logistic difficulties, thus generalization of the results of this study should be done with caution. We were only able to recruit two major ethnic groups in Malaysia, i.e. the Chinese and the Malays. Thus the results might not be suitable to be extrapolated to other ethnic minorities. This was a cross-sectional study; therefore the cause-effect relationship could not be assessed. A longitudinal study would be more appropriate in validating the relationship between SOS and TSH. The ultrasonometer used, Furuno CM-200, was only able to generate SOS as the determinant of bone health status of subjects. The other commonly used quantitative ultrasound parameter, which is the broadband attenuation (BUA) of sound, was not determined. However, previous studies had shown that SOS showed stronger association with BMD compared to BUA $(31,32)$. Other po- 
tential confounding variables, such as thyroid binding globulin and anti-thyroid antibodies were not tested in this study.

In conclusion, TSH levels were significantly associated with SOS in men after multiple adjustments. The significance of this association persisted with the inclusion or exclusion of men with subclinical hyperthyroidism. Therefore, serum TSH levels may explain some of the age-independent variation in bone health status of men. More studies are needed to validate the use of TSH as a pharmacological agent in the treatment of osteoporosis, keeping in mind the various other physiological effects of TSH on the body.

\section{Acknowledgement}

This work was sponsored by Universiti Kebangsaan Malaysia via Arus Perdana Grant (UKM-AP-TKP-09-2009), Postgraduate Research Fund (FF-376-2010) and Impak Perdana Grant (DIP-2012-07). We thank Dr. Fairus Ahmad and Dr. Elvy Suhana Mohd Ramli for performing the physical examination and medical history taking on subjects.

\section{Conflict of Interest}

\section{The authors reported no conflict of interest.}

\section{References}

1. Sachan A, Harinarayan C, Rajagopal G, Reddy P, Suresh V. Bone disease in thyrotoxicosis. Indian J Med Res. 2012; 135: 277-86.

2. Biondi B, Palmieri EA, Klain M, Schlumberger M, Filetti S, Lombardi G. Subclinical hyperthyroidism: clinical features and treatment options. Eur J Endocrinol. 2005; 152: 1-9.

3. Sun L, Liu X, Zhu L-L, Liu J, Liu Q, Iqbal J, et al. TSH and Thyroid Hormones Both Regulate Bone Mass. Clin Rev Bone Miner Metab. 2008; 6: $95-100$

4. Sendak R, Sampath TK, McPherson J. Newly reported roles of thyroid-stimulating hormone and follicle-stimulating hormone in bone remodelling. Int Orthop. 2007; 31: 753-7.

5. Baqi L, Payer J, Killinger Z, Susienkova K, Jackuliak P, Cierny D, et al. The level of TSH appeared favourable in maintaining bone mineral density in postmenopausal women. Endocr Regul. 2010; 44: 9-15.

6. Wilson S, Parle JV, Roberts LM, Roalfe AK, Hobbs FDR, Clark P, et al. Prevalence of Subclinical Thyroid Dysfunction and Its Relation to Socioeconomic Deprivation in the Elderly: A Community-Based Cross-Sectional Survey. J Clin Endocrinol Metab. 2006; 91: 4809-16.

7. Morimura T, Tsunekawa K, Kasahara T, Seki K, Ogiwara T, Mori M, et al. Expression of Type 2 Iodothyronine Deiodinase in Human Osteoblast Is Stimulated by Thyrotropin. Endocrinology. 2005; 146: 2077-84.

8. Abe E, Marians RC, Yu W, Wu X-B, Ando T, Li Y, et al. TSH Is a Negative Regulator of Skeletal Remodeling. Cell. 2003; 115: 151-62.

9. Sampath TK, Simic P, Sendak R, Draca N, Bowe AE, O'Brien S, et al. Thyroid-Stimulating Hormone Restores Bone Volume, Microarchitecture, and Strength in Aged Ovariectomized Rats*. J Bone Miner Res. 2007; 22: 849-59.

10. Sun L, Vukicevic S, Baliram R, Yang G, Sendak R, McPherson J, et al. Intermittent recombinant TSH injections prevent ovariectomy-induced bone loss. Proc Natl Acad Sci USA. 2008; 105: 4289-94.

11. Kim B-J, Lee SH, Bae SJ, Kim HK, Choe JW, Kim HY, et al. The association between serum thyrotropin (TSH) levels and bone mineral density in healthy euthyroid men. Clin Endocrinol (Oxf). 2010; 73: 396-403.

12. Kim DJ, Khang YH, Koh J-M, Shong YK, Kim GS. Low normal TSH levels are associated with low bone mineral density in healthy postmenopausal women. Clin Endocrinol (Oxf). 2006; 64: 86-90.
13. Lin J-D, Pei D, Hsia T-L, Wu C-Z, Wang K, Chang Y-L, et al. The Relationship between Thyroid Function and Bone Mineral Density in Euthyroid Healthy Subjects in Taiwan. Endocr Res. 2011; 36: 1-8.

14. Roef G, Lapauw B, Goemaere S, Zmierczak H, Fiers T, Kaufman J-M, et al. Thyroid hormone status within the physiological range affects bone mass and density in healthy men at the age of peak bone mass. Eur J Endocrinol. 2011; 164: 1027-34.

15. Grimnes G, Emaus N, Joakimsen RM, Figenschau Y, Jorde R. The Relationship between Serum TSH and Bone Mineral Density in Men and Postmenopausal Women: The Tromsø Study. Thyroid. 2008; 18: 1147-55.

16. Tauchmanovà L, Nuzzo V, Del Puente A, Fonderico F, Esposito-Del Puente A, Padulla S, et al. Reduced bone mass detected by bone quantitative ultrasonometry and DEXA in pre- and postmenopausal women with endogenous subclinical hyperthyroidism. Maturitas. 2004; 48: 299-306.

17. Krieg M-A, Barkmann R, Gonnelli S, Stewart A, Bauer DC, Del Rio Barquero L, et al. Quantitative Ultrasound in the Management of Osteoporosis: The 2007 ISCD Official Positions. J Clin Densitom. 2008; 11: 163-87.

18. Chin K-Y, Soelaiman I-N, Mohamed I, Ibrahim S, Wan Ngah W. The effects of age, physical activity level, and body anthropometry on calcaneal speed of sound value in men. Arch Osteoporos. 2012; 7: 135-45.

19. Chin K-Y, Soelaiman I-N, Mohamed IN, Ngah WZW. Serum testosterone, sex hormone-binding globulin and total calcium levels predict the calcaneal speed of sound in men. Clinics (Sao Paulo). 2012; 67: 911-6.

20. Chin K-Y, Soelaiman I-N, Mohamed IN, Mohamed N, Shuid AN, Muhammad N, et al. Discrepancy Between the Quantitative Ultrasound Value of Malaysian Men and the Manufacturer's Reference and the Impact on Classification of Bone Health Status. J Clin Densitom. 2012; in press.

21. Bonnick SL, Johnston CC, Jr., Kleerekoper M, Lindsay R, Miller P, Sherwood L, et al. Importance of precision in bone density measurements. J Clin Densitom. 2001; 4: 105-10.

22. Morris MS. The association between serum thyroid-stimulating hormone in its reference range and bone status in postmenopausal American women. Bone. 2007; 40: 1128-34.

23. Murphy E, Glüer CC, Reid DM, Felsenberg D, Roux C, Eastell R, et al. Thyroid Function within the Upper Normal Range Is Associated with Reduced Bone Mineral Density and an Increased Risk of Nonvertebral Fractures in Healthy Euthyroid Postmenopausal Women. J Clin Endocrinol Metab. 2010; 95: 3173-81.

24. Van Der Deure WM, Uitterlinden AG, Hofman A, Rivadeneira F, Pols HAP, Peeters RP, et al. Effects of serum TSH and FT4 levels and the TSHR-Asp727Glu polymorphism on bone: the Rotterdam Study. Clin Endocrinol (Oxf). 2008; 68: 175-81.

25. Vogel J. The clinical relevance of calcaneus bone mineral measurements: a review. Bone Miner. 1988; 5: 35-58.

26. Jacobs $\mathrm{C}$. The mechanobiology of cancellous bone structural adaptation. J Rehabil Res Dev. 2000; 37: 209-16.

27. Kitahara CM, Platz EA, Ladenson PW, Mondul AM, Menke A, de González AB. Body Fatness and Markers of Thyroid Function among U.S. Men and Women. PLoS ONE. 2012; 7: e34979.

28. Hase H, Ando T, Eldeiry L, Brebene A, Peng Y, Liu L, et al. TNFa mediates the skeletal effects of thyroid-stimulating hormone. Proc Natl Acad Sci USA. 2006; 103: 12849-54.

29. Baliram R, Latif R, Berkowitz J, Frid S, Colaianni G, Sun L, et al. Thyroid-stimulating hormone induces a Wnt-dependent, feed-forward loop for osteoblastogenesis in embryonic stem cell cultures. Proc Natl Acad Sci USA. 2011; 108: 16277-82.

30. Mazziotti G, Sorvillo F, Piscopo M, Cioffi M, Pilla P, Biondi B, et al. Recombinant Human TSH Modulates In Vivo C-Telopeptides of Type-1 Collagen and Bone Alkaline Phosphatase, but Not Osteoprotegerin Production in Postmenopausal Women Monitored for Differentiated Thyroid Carcinoma. J Bone Miner Res. 2005; 20: 480-6.

31. Guglielmi G, de Terlizzi F. Quantitative Ultrasond in the assessment of Osteoporosis. Eur J Radiol. 2009; 71: 425-31.

32. Töyräs J, Nieminen MT, Kröger H, Jurvelin JS. Bone mineral density, ultrasound velocity, and broadband attenuation predict mechanical properties of trabecular bone differently. Bone. 2002; 31: 503-7. 